\title{
Different Effects of Four Yogic Breathing Techniques on Mindfulness, Stress, and Well-being
}

\author{
Janika Epe ${ }^{1,}{ }^{*}$, Rudolf Stark ${ }^{1}$, Ulrich Ott ${ }^{1,2,{ }^{*}}$
}

1. Bender Institute of Neuroimaging, Justus Liebig University Giessen, Otto-Behaghel-Str. 10H, $35394 \quad$ Giessen, Germany; E-Mails: Janika.Epe@psychol.uni-giessen.de; Rudolf.Stark@psychol.uni-giessen.de; Ulrich.Ott@psychol.uni-giessen.de; ott@igpp.de

2. Institute for Frontier Areas of Psychology and Mental Health, Wilhelmstr. 3A, 79098 Freiburg i. Br., Germany

* Correspondences: Janika Epe and Ulrich Ott; E-Mails: Janika.Epe@psychol.uni-giessen.de; Ulrich.Ott@psychol.uni-giessen.de; ott@igpp.de

Academic Editor: Viann N. Nguyen-Feng

Special Issue: Yoga and Mindfulness

OBM Integrative and Complementary Medicine 2021, volume 6 , issue 3

doi:10.21926/obm.icm.2103031
Received: June 29, 2021

Accepted: September 06, 2021

Published: September, 18, 2021

\begin{abstract}
Yogic breathing techniques are fundamental to the physical and mental practice of yoga. They are closely connected to meditation, which involves the observation of breath. There are many yogic techniques based on the active regulation of the breath. Breathing practices influence many processes in the body, e.g. heart rate variability, and the mind, e.g. relaxation and stress, through their impact on the autonomic nervous system. This study intended to investigate differential effects of four yogic breathing techniques: (1) ujjayi-relaxation through slowing down the breath, (2) paced breathing-enhancement of concentration by following a precise protocol of slowdown breathing, (3) kapalabhati-raising wakefulness by mild hyperventilation, and (4) alternate nostril breathing-balancing the autonomic nervous system by alternating breath between the two nostrils. This study was conducted on 36 participants, who learned each technique within two weeks of an eight-week program and practiced them daily. After each technique, mindfulness, perceived stress, and physical well-
\end{abstract}

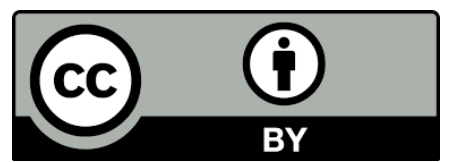

(C) 2021 by the author. This is an open access article distributed under the conditions of the Creative Commons by Attribution License, which permits unrestricted use, distribution, and reproduction in any medium or format, provided the original work is correctly cited. 
being were assessed based on questionnaires. Ujjayi breathing, showed a relaxing effect, reduced stress, increased peacefulness, and the feeling of being at ease/leisure. Paced breathing resulted in a greater awareness of inner experiences. Kapalabhati showed a significant increase in vitality and joy of life, and alternate nostril breathing showed no hypothesis-compliant changes. The findings of this study suggest several beneficial and differential effects of these breathing techniques; therefore, they could be employed as tools for self-regulation in therapeutic contexts.

\section{Keywords}

Breathing; yoga; self-regulation; ujjayi; paced breathing; kapalabhati; alternate nostril breathing

\section{Introduction}

The act of breathing is an essential aspect of most meditative practices. Most forms of meditation involve focusing on an object, which can be one's breath [1]. Therefore, breath serves as an anchor for attention, such as focusing on the movement of the abdomen while breathing or observing the respiratory flow throughout the body [1]. From a scientific perspective, breathing techniques can be conceived as tools for self-regulation because they reliably elicit specific physiological responses. Also, conscious breathing can explain many positive effects of meditation, e.g., its physiological effects on the autonomic nervous system [2].

In Buddhist meditation, passive observation of the breathing process is typically used. However, in yogic practices, we find many methods based on the active regulation of the breathing process, subsumed under the Sanskrit term pranayama (prana: breath, vital energy; ayama: extending, controlling). Within yoga, the techniques of pranayama are part of preparatory exercises meant to calm down the mind and prepare it for concentration and meditation. The last of the five verses dealing with pranayama in the Yoga-Sutra reads: "II.53 And [the yogin gains] the fitness of the mind for concentration" (p. 93, [3]).

The beneficial effects of different yogic breathing techniques have been studied extensively. In their review article, Saoji, Raghavendra, and Manjunath (2019) found breathing practices to be able to "influence the neurocognitive abilities, autonomic and pulmonary functions as well as the biochemical and metabolic activities in the body" (p. 57, [4]). However, the diversity of breathing techniques in yoga is striking, which may be the reason behind its varying physiological and psychological effects. There are techniques to slow down (e.g., ujjayi, paced breathing and holding the breath) or to accelerate the breathing rate (bhastrika and kapalabhati), techniques involving the production of humming sounds (brahmari), tongue positions to modify the airflow (sitali), and the occlusion of nostrils for unilateral or alternate nostril breathing (nadi shodana, anuloma viloma).

In our study, we compared four prominent yogic breathing techniques (ujjayi, paced breathing, kapalabhati, and alternate nostril breathing) with respect to their differential effects on mindfulness, stress, and well-being. We aimed at evaluating the potential of these four different techniques to facilitate relaxation, reduce stress, enhance concentration and wakefulness, and balance the arousal level. 
One of the most studied techniques for relaxation and stress reduction is slowing down the breathing rhythm; this technique serves as a connection between all other relaxation techniques [5]. Within yogic breathing, ujjayi is a commonly used technique to slow down the breathing rhythm and make practitioners aware of their breathing patterns during the practice of yogic postures (asana). During this technique, vocal cords are constricted, similar to whispering, so that airway resistance rises leading to the production of a soft sound within the throat by the airflow [6].

Controlled deep breathing is described as one of the oldest and most efficient methods to reduce acute stress as it is known to regulate vegetative functions, thereby altering our mental state [7]. A review by Zaccaro et al. showed that slow breathing has a positive effect on comfort, pleasantness, relaxation, anxiety, depression, anger, and confusion as well as reduces the symptoms of arousal [8]. It is also described as an effective non-pharmacological intervention to control emotions [9].

Another, more demanding approach for slowing down the breathing rate is the paced breathing method in combination with holding the breath (kumbhaka). These techniques divide one breathing cycle into four phases: (1) inhalation, (2) pause after inhalation, (3) exhalation, and (4) pause after exhalation. The duration of each phase is determined by counting slowly, for example, with the help of fingertips or knuckles, or with sound guidance. However, these techniques demand a high level of concentration from the practitioner.

A slow breathing frequency (about $10 \mathrm{~s}$ per breath) is known to increase baroreflex sensitivity and heart rate variability (HRV), which are the markers of intact vegetative regulation [10]. The connection between HRV and breathing lies in the change of heart rate with respiration, the socalled respiratory sinus arrhythmia (RSA). During inhalation, the heartbeat accelerates, and during exhalation, it slows down. A higher difference in the heart rate during inhalation and exhalation results in a higher RSA and HRV, which stands for better flexibility of autonomic regulation. Biofeedback-trainings showed that increasing HRV is mainly achieved through slow and even breathing [11].

Another approach to regulating the breath is to accelerate the breathing rhythm as in hyperventilation, which activates the central nervous system and shifts blood gas levels (hypocapnia). In the yogic tradition, these methods are known as "cleaning" techniques. They are called bhastrika and kapalabhati and work with the active movement of the abdominal muscles. Kapalabhati focuses on an active exhalation; the muscles of the abdomen are tensed quickly, causing the abdomen to retract and the air to flow out of the lungs. The muscles are then relaxed again, allowing air to passively flow into the lungs. In bhastrika, during exhalation, the abdomen is tensed quickly and during inhalation, the diaphragm is tensed; this leads to an active expansion of the lungs, thereby enforcing the airflow during the process of breathing in and out [12].

The activating effect of hyperventilation was proved by electroencephalogram (EEG)-studies [13]. This activation of the central nervous system has found several applications, e.g., it is used to provoke epileptic seizures for diagnostic purposes [14]. The hyperventilation during kapalabhati is rather mild because of the pauses between phases of fast breathing. Therefore, it is not surprising that studies comparing groups practicing slow versus fast breathing techniques found significant physiological effects in the slow breathing group, such as increased parasympathetic activity, decreased sympathetic activity, and changes in cardiovascular variables, but not in the fast breathing group $[15,16]$. However, on a subjective level, both groups reported reduced perceived stress after the intervention [16]. Nevertheless, we expect kapalabhati to have an activating effect due to the fast muscular movements and increased ventilation of the lungs when compared to other 
breathing techniques. An activating effect on a psychological level might be reflected in higher feelings of vitality and an enhanced ability to concentrate and react on a cognitive level.

Finally, we investigated the balancing effect of the alternate nostril breathing method (nadi shodhana, anuloma viloma). During this breathing technique, one nostril is occluded so that the breath flows only through the other nostril. A distinction is made between unilateral nostril breathing (right or left nostril occluded for the entire duration of the practice) and alternate nostril breathing (one breath through the left nostril then through the right by alternately closing the other nostril).

Studies on the unilateral nostril breathing technique found varying effects on blood pressure depending on the nostril used [17]. Breathing through the left nostril had a sedative effect, while breathing through the right nostril had an activating effect. Alternate nostril breathing was found to have a balancing effect on the vegetative nervous system, probably modulated by the balanced breathing rhythm. It also had an effect on the lateralization of EEG activity between the left and right hemispheres [18]. Telles, Verma, Sharma, Gupta, and Balkrishna found positive effects of the alternate nostril breathing method on blood pressure, attention, and cognitive performance [19].

The physiological changes caused by the breathing techniques also lead to different psychological effects. One example is the connection between HRV and the brain structures that are known to regulate emotions and manage stress [20]. The significance of breathing techniques as a regulatory mechanism is because of the direct modification of vegetative processes that otherwise run autonomously. Therefore, by practicing breathing techniques, automatic bodily processes can be influenced willfully.

The findings of the above-mentioned studies point to the positive effects of breathing techniques and suggest that different techniques have different effects on psychological and physiological variables. Although a growing number of studies on the effects of different yogic breathing techniques have been published in recent years, a study comparing the effects of different breathing techniques on the same subjects has not been conducted yet. There is a possibility of marked differences in the effects of slow, fast, and balanced breathing techniques on the same subjects. Within-subject designs are better able to identify differential effects because intersubjective variability is excluded. In addition, we wanted the subjects to learn all techniques in order to study the differences in preference and application. In this study, we asked the participants whether and how they used the learned techniques in everyday life with a self-constructed questionnaire, immediately after the training and also six months later. Our objective was to determine whether our participants integrated the breathing techniques for self-regulation in their daily routine (for calming, concentrating, activating, and balancing the mind) and whether differences regarding usability existed between the four breathing techniques.

\subsection{Hypotheses}

We expect that the breathing techniques will have differential effects on the subjective states reported by the participants. Our hypotheses are based on the following reported effects of the individual breathing techniques: (1) promotes relaxation, (2) increases concentration, (3) enhances activation, and (4) balances arousal. 
Hypothesis 1: After practicing slow breathing (ujjayi), we expect significantly reduced perceived stress as well as an enhanced feeling of peacefulness and being at ease/leisure, and pleasant tiredness in the participants compared to that of the baseline level, i.e., before the training.

Hypothesis 2: After following the precise protocol for slowdown breathing (paced breathing), we expect significantly higher awareness of internal experiences and more ability to concentrate and react in the participants compared to that of the preceding breathing technique.

Hypothesis 3: After mild hyperventilation (kapalabhati), we expect significantly higher vitality, the joy of life, concentration, and reaction in the participants compared to that of the preceding breathing technique.

Hypothesis 4: We expect that the balancing of the vegetative nervous system after practicing alternate nostril breathing (nadi shodhana) leads to a mental state of contentment, accompanied by a significantly stronger non-judgmental attitude and greater satisfaction with current body condition in the participants compared to that of the preceding breathing technique.

\section{Materials and Methods}

\subsection{Participants}

Participants, consisting of students and employees, were recruited using the university mailing list. The number of subjects was determined by practical constraints (room and time) for conducting the training and due to the exploratory nature of the study, prior power analysis of the participants was not carried out. Thirty-six participants started the measurements (18 male, 18 female, mean age: $28.6, \mathrm{SD}=8.9$, and range 21-63); 32 completed the whole training. Two participants participated in only three baseline measurements and two measurements with a breathing practice; two women quit after the baseline measurement because of time issues. Twenty-six subjects (16 male, 10 female, mean age: 29.7, SD $=9.9$, and range 21-63) participated in a follow-up measurement after six months. The other participants did not respond to the invitation of followup measurement or could no longer arrange a measurement due to relocation. Exclusion criteria were the diagnosis of epilepsy (also in first-degree relatives), lung dysfunction, acute psychotherapeutic treatment, and experience with yogic breathing techniques.

\subsection{Procedure}

For the data collection, a multiple baseline design was employed [21]. Participants were randomly assigned to three groups of twelve persons each, and they started the training at three different time points. The training of the different breathing techniques is referred to as "intervention" because we conceptualized them as active self-regulation techniques that could be applied by the participants themselves in order to change their mental state as desired.

All participants took part in the first baseline measurement (week one), and twelve participants immediately started the training (first intervention: ujjayi; group 1). After two weeks of training or waiting, all participants were measured once again, either for their second baseline measurement (groups 2 and 3) or for an intervention measurement (group 1, week four). Afterward, the twelve participants from group 1 continued the training with one of the other three different interventions (they were randomly assigned to a specific sequence of the remaining three interventions; four participants per training session), and another twelve started the training (group 2) with the first 
intervention (ujjayi). After another two weeks of training, all participants were measured again (group 3: baseline; groups 1 and 2: intervention measurements, week seven). After that, the third group started the training too with the first intervention (ujjayi); the others continued with one of the other three different interventions (members of group 2 had their training sessions together with members of group 1). Every time there were two weeks of training and one week of measurements, in which all participants were measured once. Therefore, group 1 had one baseline measurement before the training started, group 2 had two baseline measurements, and group 3 had three baseline measurements before the training started.

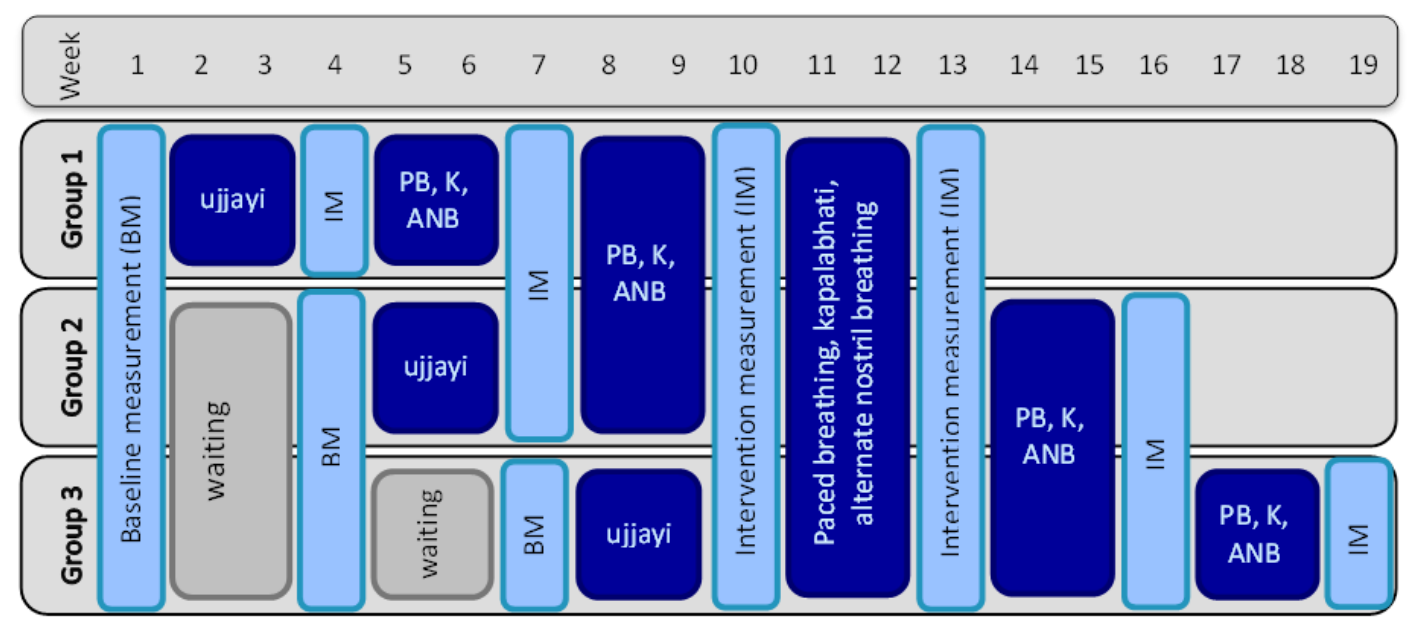

Figure 1 The training procedure of the three groups, including measurement time points. Baseline measurements (BM) and intervention measurements (IM) are marked. The first intervention, ujjayi, is shown separately from the other three interventions, i.e., paced breathing $(\mathrm{PB})$, kapalabhati $(\mathrm{K})$, and alternate nostril breathing (ANB).

The breathing training was an eight-week program (not counting the measurement weeks between the two-week training blocks) with four modules of a yogic-breathing-technique training. Each breathing technique was taught in two weeks, with group sessions, additional video-learning material, and worksheets. The 60 min sessions were held with the presence of four to twelve participants in a yogic classroom. The instructor had undergone training for two years to become a hatha yoga teacher and had one and a half years of teaching and ongoing supervision experience. Most postures were taught during the first intervention; only small adjustments were made during the other interventions as more focus was given on the teaching of the new breathing technique. The description of the breathing training was published as a book (in German) [22]. The audio files used for paced breathing and the video tutorials showing the asanas taught can be downloaded free from https://sites.google.com/site/gesunddurchatmen.

The first intervention (ujjayi) included four group sessions; all others included two group sessions and two online tutorials. During the first intervention, participants had two group sessions per week; the other interventions included one group session and one online tutorial per week. Participants had a structured plan with a daily routine to practice until the next session. Every exercise that they learned in the sessions or tutorials was available online as tutorial videos. Participants were required to practice their breathing techniques daily and record their practice. The training always started with the same breathing technique (ujjayi); the other three techniques followed in different 
sequences (fully permuted across all participants). As mentioned previously, the training sessions were $60 \mathrm{~min}$ long; the daily routine for their home practice took about $20 \mathrm{~min}$ to complete.

\subsubsection{Ujjayi}

This intervention formed the basis of the program, in which participants learned yogic postures, felt their breath in different parts of the body, combined movement-flows with the breathing rhythm, and learned the ujjayi-breathing technique. It included four group sessions and video-based training between the sessions.

Participants were made to learn how to feel their body, their movements, and their breathing, and bring them all together. They were instructed to get to know and accept their boundaries and adapt the exercises to their ability level, i.e., being aware of themselves without evaluating. With the possibility of adaptation, it was ensured that they had a regular and steady breathing rhythm, i.e., not too fast, or unsteady, which may be caused by overextension.

By practicing this technique, the participants learned how to use the movement of their breath in the body to support postures and movements. One of the underlying principles was to differentiate between movements resulting in a reduced volume of the lungs and movements resulting in increased volume of the lungs ("opening" and "closing" movements). The goal was to find and perform the movements in a way so that the breath can adapt its rhythm and flow freely.

After the participants learned the yogic movement principles and became aware of the interactions between their body and breath, they learned ujjayi (for a description, see introduction).

\subsubsection{Paced Breathing}

In this intervention, participants learned to prolong their breathing cycle based on audio files. They became aware of the length of inhalation and exhalation and the pause in between (four phases of breathing: inhalation-holding-exhalation-holding). The audio files guided the participants in prolonging their breath at the ratio of 1:2 or 2:3 of inhalation and exhalation (see Table 1 and Table 2). Participants used a ratio that was closer to their natural breathing rhythm. Files were created with the program "Audacity" (Version 2.1.2; The Audacity Team, 2016). Based on a study of paced breathing, we employed a frequency of $330 \mathrm{~Hz}$ for inhalation and $262 \mathrm{~Hz}$ for exhalation, respectively [23].

Table 1 Duration of individual breathing phases in seconds and total length of audio files in minutes, including eight repetitions. Converted breathing ratio: 1:2.

\begin{tabular}{lllll}
\hline Inhalation & Holding & Exhalation & Holding & Length \\
\hline 03 & 00 & 06 & 00 & $1: 12$ \\
03 & 00 & 06 & 02 & $1: 28$ \\
03 & 02 & 06 & 02 & $1: 44$ \\
04 & 02 & 08 & 02 & $2: 08$ \\
04 & 03 & 08 & 03 & $2: 24$ \\
05 & 03 & 10 & 03 & $2: 48$ \\
06 & 04 & 12 & 04 & $3: 28$ \\
\hline
\end{tabular}


Table 2 Duration of individual breathing phases in seconds and total length of audio files in minutes, including eight repetitions. Converted breathing ratio: 2:3.

\begin{tabular}{lllll}
\hline Inhalation & Holding & Exhalation & Holding & Length \\
\hline 04 & 00 & 06 & 00 & $1: 20$ \\
04 & 00 & 06 & 02 & $1: 36$ \\
04 & 02 & 06 & 02 & $1: 52$ \\
06 & 00 & 09 & 00 & $2: 00$ \\
06 & 00 & 09 & 03 & $2: 24$ \\
06 & 03 & 09 & 03 & $2: 48$ \\
06 & 04 & 09 & 04 & $3: 04$ \\
\hline
\end{tabular}

\subsubsection{Kapalabhati}

In this intervention, participants learned how to accelerate the breath and pause it. They practiced a mild and controlled form of hyperventilation, followed by a spontaneous pause in breathing. After they learned to pay specific attention to the movement of the breath in the abdomen, they were asked to focus on the moment after exhalation. They were instructed not to inhale automatically, but to wait until their body gave them the impulse for inhalation, resulting in a longer pause between exhalation and inhalation. Afterward, they learned how to intensify exhalation by tensing the abdomen at the end of the exhalation and relaxing it consciously before the inhalation, so the air could flow into the lungs; this resulted in an active exhalation and passive inhalation. By practicing this, they learned how to increase their breathing rhythm up to one breath per second. They took 20-60 breaths per minute, increasing the number slowly based on their level of experience.

\subsubsection{Alternate Nostril Breathing}

During this intervention, participants learned the "alternating nostril breathing" technique. They started by taking one breath (exhale-inhale) through both nostrils, then closing the right nostril with the thumb of the right hand (in the pause between inhalation and exhalation) and breathing out and in through the left nostril. After breathing in, they closed the left nostril with the ring finger and opened the right one, breathed out and in, and closed the right nostril and opened the left one to breathe out and breath in again. In this way, they took 16-32 breathes, increasing the number slowly based on their level of experience.

\subsection{Measurements}

For the baseline measurements, participants sat in a chair in a dark room with a small light and closed eyes. For $10 \mathrm{~min}$, they were left alone without any instructions (resting state) and some physiological recordings were made (not reported here). Afterward, participants completed a computer-based attention test and different questionnaires.

In intervention measurements, the effect of the intervention learned in the previous two weeks was examined. Similar to the baseline measurements, participants sat in a chair in the same room with similar environmental conditions; the breathing rhythm was recorded to verify if a breathing 
technique was used. First, they were asked to sit still with their eyes closed for 5 min to record the baseline measurement. Afterward, they were instructed to perform the breathing technique for 10 min. During this time, the physiological parameters were measured again. Then, the computer test was administered, and questionnaires were filled out, referring to the last two weeks. This article focuses on psychological variables, which were assessed by the following questionnaires: Comprehensive Inventory of Mindfulness Experiences (CHIME) [24], the Perceived Stress Questionnaire (PSQ) [25], and a questionnaire on the positive aspects of current physical well-being (FAW) [26] (for further information, see Materials).

At the end of their last intervention measurement, participants completed a questionnaire about all interventions and the benefit of using them in everyday life. They also completed a similar questionnaire in a follow-up measurement after six months.

\subsection{Materials}

To investigate the subjective effect of the breathing techniques on mindfulness, stress, and wellbeing, the participants were made to complete three questionnaires after the training of each breathing technique.

CHIME is a psychometrically validated instrument for the self-assessment of mindfulness [24]. It covers different aspects of mindfulness. The eight implemented scales are (1) awareness of internal experiences, (2) awareness of external experiences, (3) acting with awareness, (4) accepting nonjudgmental attitude, (5) nonreactive decentering, (6) openness to experience, (7) awareness of thoughts' relativity, and (8) insightful understanding [27]. Items are rated on a six-point Likert scale, from (1) almost never to (6) almost always. For score calculation, scores of the items are summed and divided by the number of items of the respective (sub-) scale. Higher scores correspond to a higher value of mindfulness in that aspect. For our purpose, we used the scales: (1) Awareness of internal experiences and (4) accepting non-judgmental attitude. These scales were closely connected to the experiences of mindfulness during the yoga classes and seemed to be indicative of the differential effects of the breathing techniques.

The stress experience of the participants was assessed with the validated German version of the PSQ [25]. Items are rated on a four-point Likert scale, from (1) almost never to (4) almost always. For score calculation, scores of the items are summed and divided by 30 (number of items); one was subtracted from the value and then score was divided by three to get a number between zero and one.

Well-being was assessed with a questionnaire (FAW) on the positive aspects of the current physical well-being [26]. The questionnaire comprises seven factors, which are assessed with 58 items. The seven sub-scales are (1) satisfaction with the current state of the body, (2) peacefulness and being at ease/leisure, (3) vitality and joy of life, (4) decreasing tension/pleasant tiredness, (5) enjoyment/pleasure, (6) ability to concentrate and react, and (7) well-groomed, fresh, and pleasant body sensation. Items are rated on a five-point Likert scale, from (0) never to (4) entirely. For score calculation, item scores are summed up. In this study, we used the scales: (1) Satisfaction with the current state of the body, (2) peacefulness and being at ease/leisure, (3) vitality and joy of life, (4) decreasing tension/pleasant tiredness, and (6) ability to concentrate and react. These scales seemed to describe possible bodily experiences during the yogic breathing training. 
The final questionnaire was a self-developed instrument. The subjects were asked to rate the possible effects of breathing training and its usefulness in everyday life. Questions were phrased in the first-person perspective and rated on a scale from one (strongly disagree) to six (strongly agree). Examples of formulations are as follows: I found the training to be...a) balancing, b) activating, c) relaxing, d) concentration enhancing; I was able to incorporate the breathing techniques into my everyday life...a) ujjayi, b) paced breathing, c) kapalabhati, d) alternate nostril breathing.

\subsection{Data Analysis}

Data were analyzed using IBM SPSS Statistics 27. Scores of the questionnaires and scales were calculated considering negatively coded items. In order to exclude a sole time effect, baseline measurements were first compared with each other, calculating univariate analysis of variance (ANOVA) with repeated measures. The measurements of each intervention were compared with the measurements taken before the intervention (i.e., two weeks before). Due to the randomized allocation of the sequence of interventions (except first intervention, i.e., ujjayi breathing), this form of analysis was intended to exclude the effects of previous interventions (apart from the baseline intervention that always took place first). Univariate ANOVAs with repeated measures were calculated. The hypotheses were tested on a five percent significance level. The final questionnaire was analyzed on an exploratory basis.

The study was ethically approved by the ethics committee of the Faculty of Psychology and Sport Science of the Justus Liebig University Giessen, Germany. The identification code was 2017-0002 (date of approval: 05.04.2017), and the study followed the German national legislation. Written informed consent was obtained from all participants.

\section{Results}

Analyses of practice times showed that there were differences in the duration for which different breathing techniques were practiced, with ujjayi being practiced the most. The ujjayi intervention was practiced at home (about 20 min practicing time) at a frequency of around ten times in two weeks, whereas the other interventions were practiced at home for seven to eight times in two weeks (ujjayi: $M=10.3, S D=2.5$; paced breathing: $M=8.0, S D=2.8$; kapalabhati: $M=7.4, S D=4.7$; alternate nostril breathing: $M=7.8, S D=3.3 ; F[3,45]=4.93, p=0.01)$. There were no significant differences in practice time between the three breathing techniques, excluding ujjayi $(F[2,30]=$ $0.039, p=0.68$ ).

To investigate training-independent effects, baseline measurements were compared. Analysis of the used scales of the CHIME showed no significant differences between the three baselinemeasurements ([1] awareness of internal experiences: $F[2,20]=0.419, p=0.66$; [4] accepting nonjudgmental attitude: $F[2,20]=0.96, p=0.4)$. Comparison of baseline measurements regarding the PSQ stress score showed no significant differences between the three points in time $(F[2,18]=1.03$, $p=0.38)$. Also, comparison of the scales of the FAW showed no significant differences in baseline conditions ([1] satisfaction with current state of body: $F[2,20]=1.20, p=0.32$; [2] peacefulness and being at ease/leisure: $F[2,20]=1.25, p=0.31 ;[3]$ vitality and joy of life: $F[2,20]=1.74, p=0.2 ;[4]$ decreasing tension, pleasant tiredness: $F[2,20]=2.47, p=0.11$; and $[6]$ ability to concentrate and react: $F[2,20]=0.75, p=0.48)$. 
To test hypothesis 1 , the results after the practice of ujjayi were compared with the values from the baseline measurements. Results of perceived stress showed a significant reduction after two weeks of learning and practicing ujjayi $(F[1,33]=3.97, p=0.05)$. Participants reported significant increase in the parameters of peacefulness and being at ease/leisure $(F[1,33]=7.03, p=0.01)$ and decreasing tension/pleasant tiredness $(F[1,33]=27.63, p<0.001)$. Participants reported a significantly higher accepting non-judgmental attitude $(F[1,31]=13.86, p \leq 0.001)$, which was not predicted by our hypothesis.

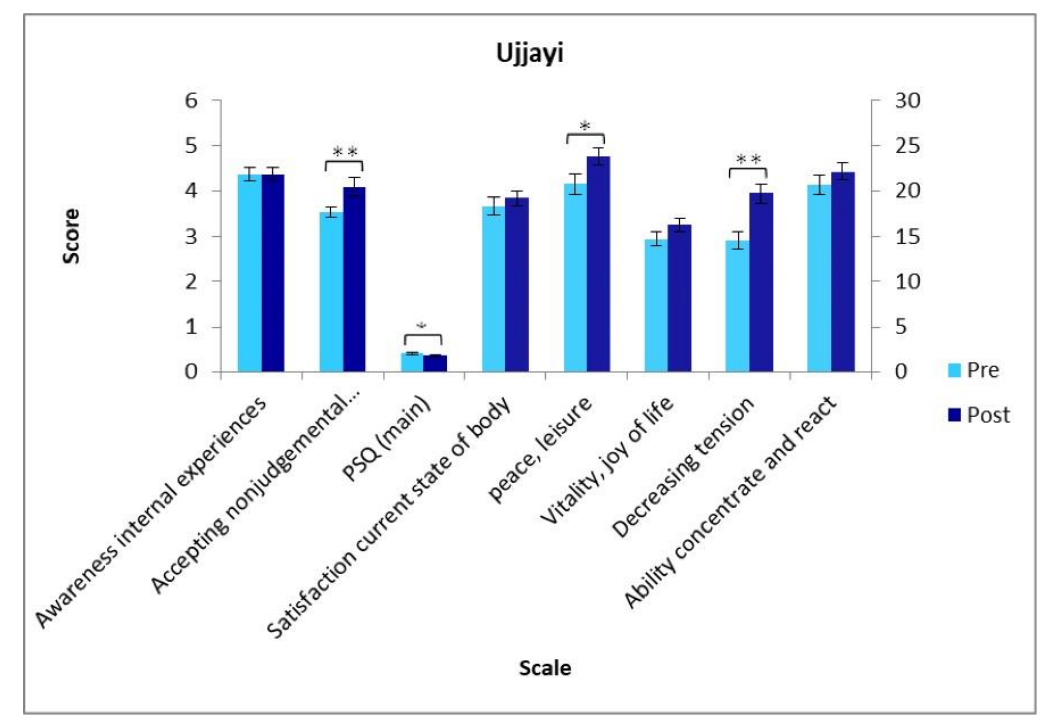

Figure 2 Mean score and standard errors of data referring to ujjayi breathing technique (Post) and the preceding measurement (Pre). Scales referring to three questionnaires: CHIME (awareness of internal experiences, accepting non-judgmental attitude) and PSQ (mean score) are mapped on the left y-axis. Scores from FAW are mapped on the right $y$-axis (satisfaction with current state of body, peacefulness and being at ease/leisure, vitality and joy of life, decreasing tension/pleasant tiredness, and ability to concentrate and react). Significant results are marked $(* p \leq 0.05, * * p \leq 0.001), \mathrm{n}=32-34$.

Hypothesis 2, referring to the paced breathing technique, with the precise slowdown of breath, was tested by comparing the values after two weeks of training with the values of the measurement right before this intervention (measurement referring to one of the other breathing techniques). Results showed a significant increase in awareness of internal experiences $(F[1,31]=6.49, p=0.02)$ but no significant changes were observed in the ability to concentrate and react $(F[1,31]=0.72, p$ $=0.4)$. 


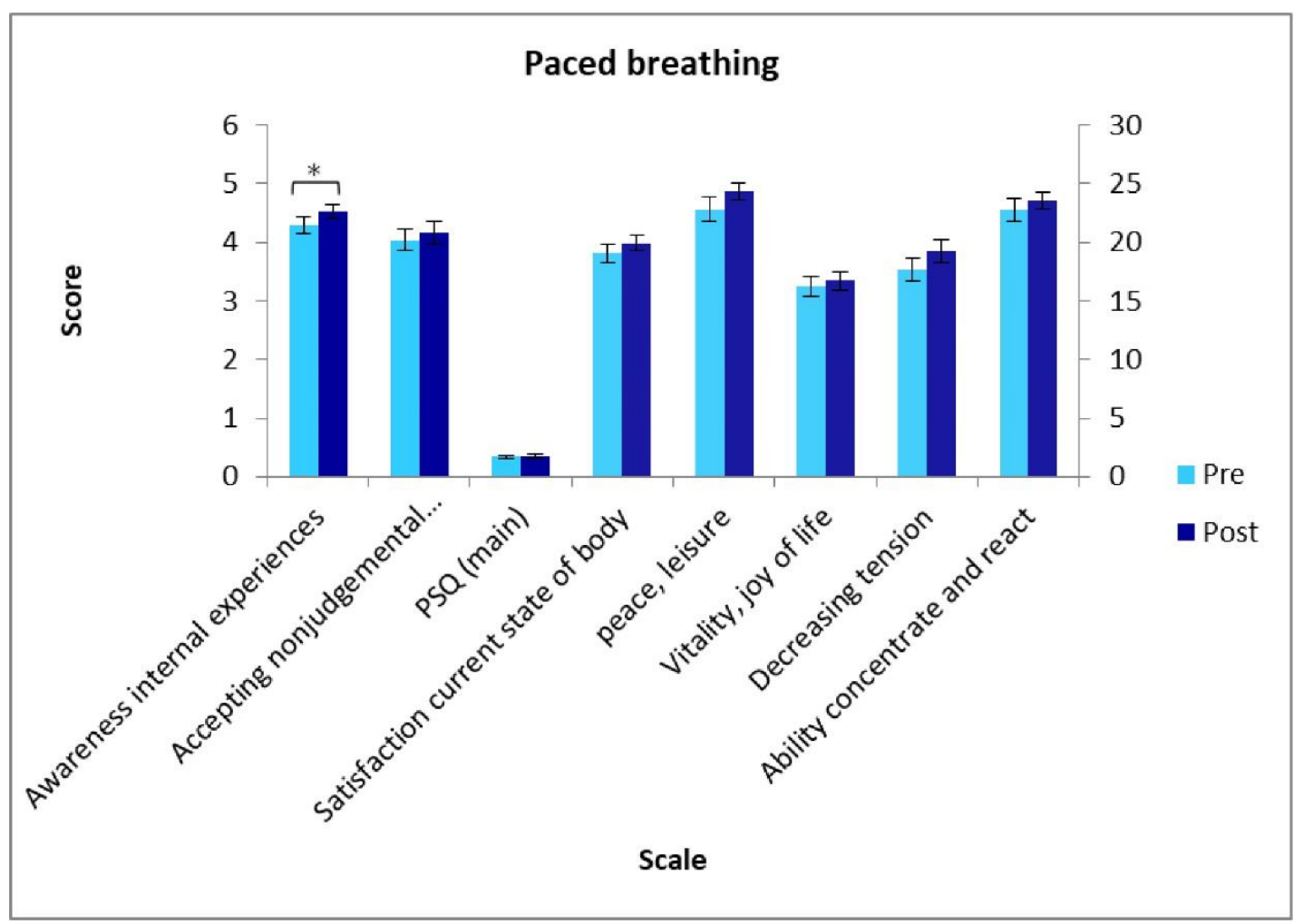

Figure 3 Mean score and standard error referring to the paced breathing technique (Post) and the preceding measurement (Pre). Scales referring to three questionnaires: CHIME (awareness of internal experiences and accepting non-judgmental attitude) and PSQ (Mean score) are mapped on the left y-axis. Scores from FAW are mapped on the right $y$-axis (satisfaction with current state of body, peacefulness and being at ease/leisure, vitality and joy of life, decreasing tension/pleasant tiredness, and ability to concentrate and react). Significant results are marked $\left({ }^{*} p \leq 0.05,{ }^{* *} p \leq 0.001\right), \mathrm{n}=32-34$.

Hypothesis 3 predicts changes after practicing kapalabhati and was tested by comparing values after two weeks of training with values of the measurement before this intervention (measurement referring to one of the other breathing techniques). After practicing kapalabhati, participants described significant increase in vitality and joy of life $(F[1,32]=4.90, p=0.003)$, but no significant changes were observed in subjective ability to concentrate and react $(F[1,32]=2.55, p=0.12)$. 


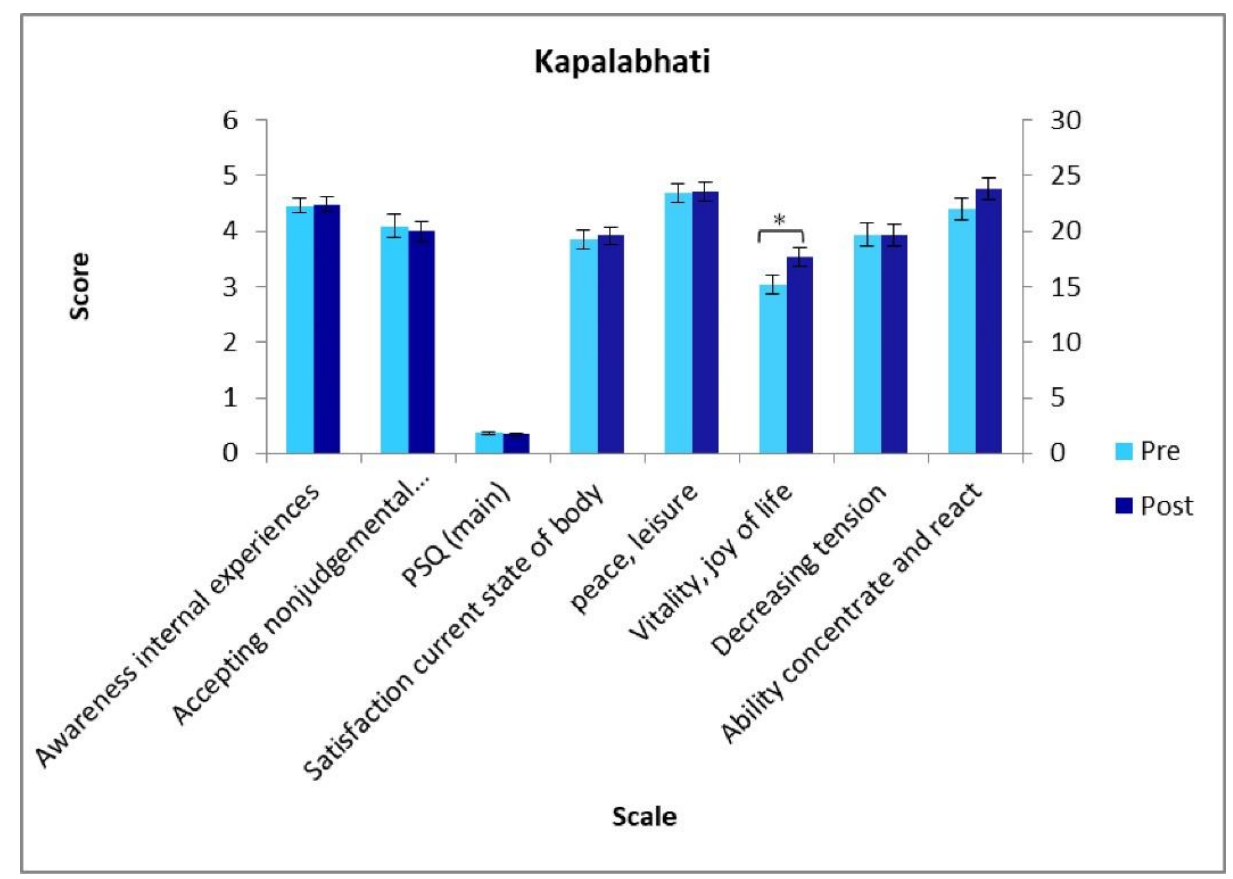

Figure 4 Mean score and standard error referring to the practicing of kapalabhati (Post) and the preceding measurement (Pre). Scales referring to three questionnaires: CHIME (awareness of internal experiences and accepting non-judgmental attitude) and PSQ (Mean score) are mapped on the left y-axis. Scores from FAW are mapped on the right $y$-axis (satisfaction with current state of body, peacefulness and being at ease/leisure, vitality and joy of life, decreasing tension/pleasant tiredness, and ability to concentrate and react). Significant results are marked $\left({ }^{*} p \leq 0.05,{ }^{* *} p \leq 0.001\right), n=32-34$.

Hypothesis 4, referring to changes after practicing alternate nostril breathing, was tested by comparing the measurement after two weeks of training with values of the measurement before this intervention (measurement referring to one of the other breathing techniques). Participants showed no significant changes in accepting non-judgmental attitude $(F[1,30]=2.43, p=0.13)$ and satisfaction with current state of body $(F[1,33]=1.14, p=0.29)$. Participants reported a reduced vitality and joy of life after practicing alternate nostril breathing $(F[1,31]=4.68 ; p=0.04)$, which was not predicted in our hypothesis. 


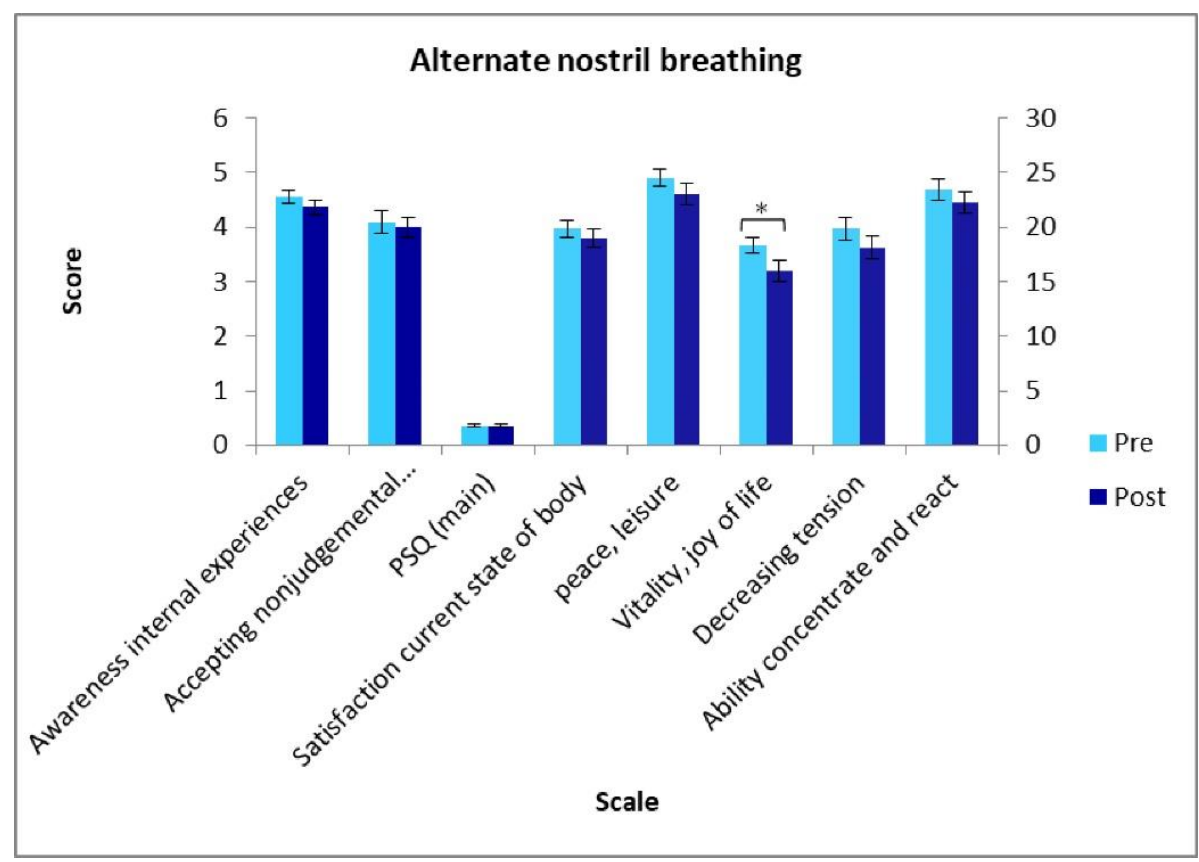

Figure 5 Mean score and standard error referring to the practicing of alternate nostril breathing (Post) and the preceding measurement (Pre). Scales referring to three questionnaires: CHIME (awareness of internal experiences, accepting non-judgmental attitude) and PSQ (Mean score) are mapped on the left y-axis. Scores from FAW are mapped on the right y-axis (satisfaction with current state of body, peacefulness and being at ease/leisure, vitality and joy of life, decreasing tension/pleasant tiredness, and ability to concentrate and react). Significant results are marked ( $\left.{ }^{*} p \leq 0.05, * * p \leq 0.001\right)$, $n=32-34$.

Overall, the training was very calming $(M=5.77, S D=0.42)$, enhanced the concentration $(M=$ 4.97, $S D=0.91)$, and balanced the mind $(M=5.65, S D=0.55)$ of the subjects. Among the effects, the activating effect received the lowest level of agreement from the subjects; however, for this effect, they gave the response of "more likely to agree" ( $M=4.68, S D=1.14)$. After six months of the training, similar tendencies were observed in the responses of the participants. Highest values were received for the balancing $(M=5.39, S D=0.72)$ and calming $(M=5.35, S D=0.93)$ effect of the training; the agreements for the concentrating $(M=4.57, S D=0.73)$ and activating $(M=4.39, S D=$ 1.2 ) effect was not that strong but was still reported. 


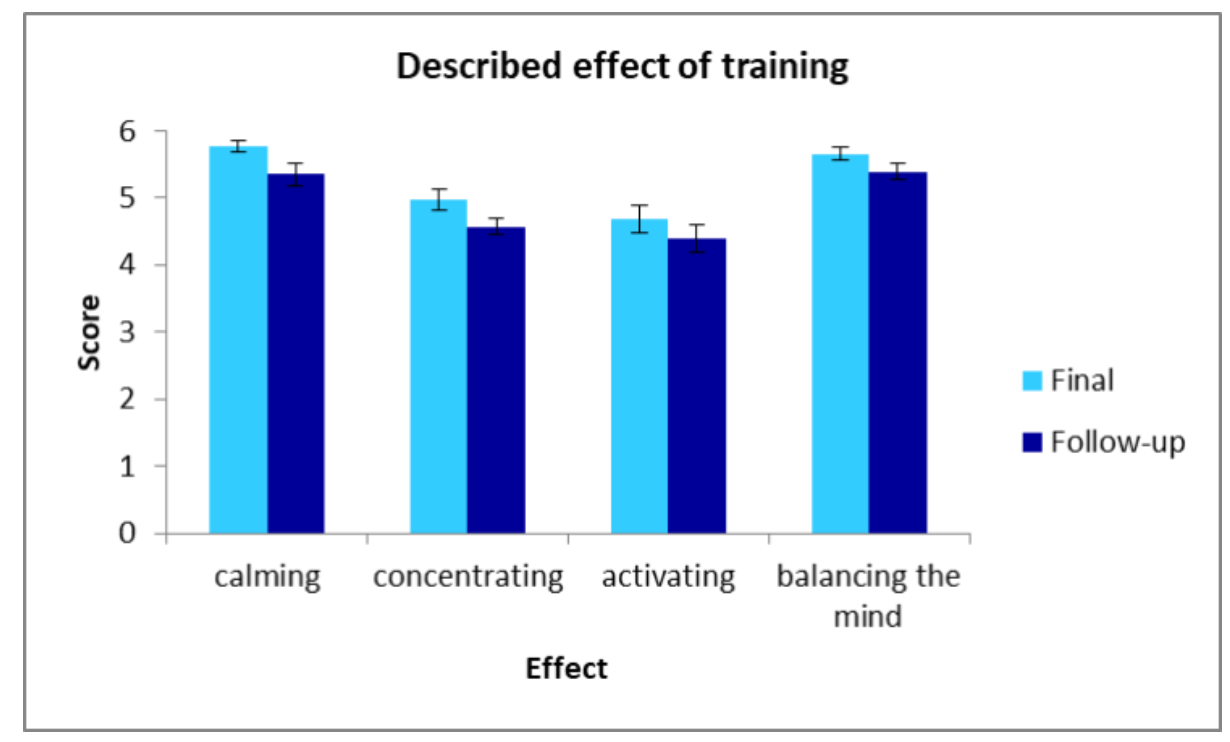

Figure 6 Mean score and standard errors of the described effects of the training in the final questionnaire (Final) and the follow-up measurement after six months (Follow-up), $n=32 / 26$.

When we look at the integration of breathing techniques into everyday life, ujjayi was the most practiced technique $(M=4.48, S D=1.16)$, even after 6 months $(M=4.04, S D=1.82)$. Also, $54.8 \%$ of the participants described ujjayi as the easiest to learn, while $19.4 \%$ described the paced breathing intervention as the easiest to learn. On average, participants described paced breathing as moderately easy to implement in everyday life $(M=3.58, S D=1.92)$. The follow-up measurement showed that the breathing techniques except ujjayi were difficult to implement (paced breathing: $M=2.43, S D=1.8$; kapalabhati: $M=1.68, S D=1.13$; and alternate nostril breathing: $M=2.43, S D=$ 1.41). Also, immediately after the training, kapalabhati and the alternate nostril breathing were described as "merely useful" (kapalabhati: $M=3.32, S D=1.81$; alternate nostril breathing: $M=3.39$, $S D=1.65)$. Kapalabhati was described as the most difficult to learn by $48.4 \%$ of the participants.

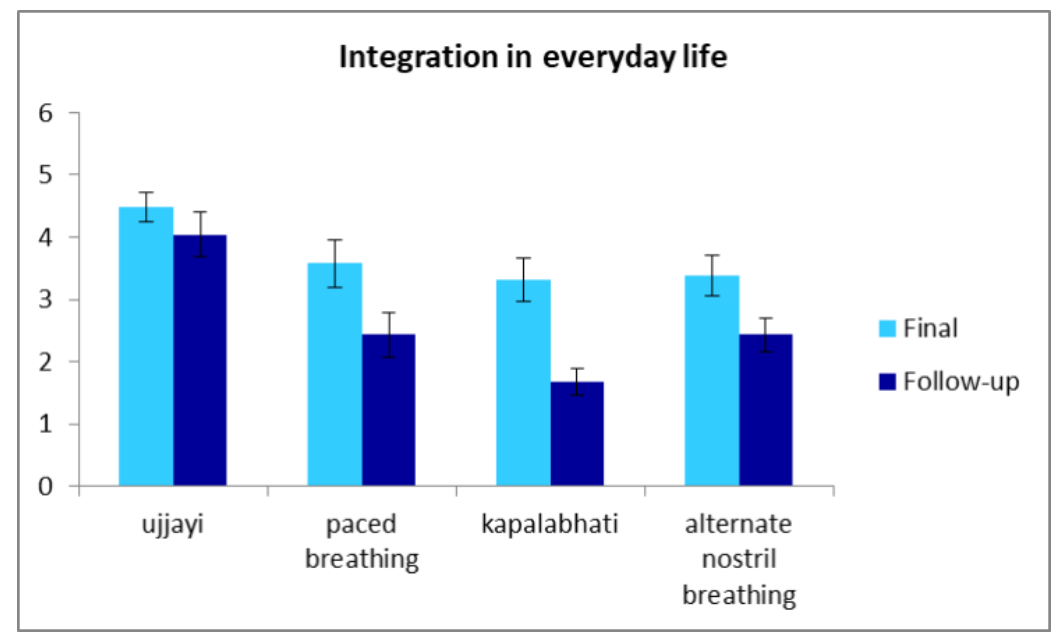

Figure 7 Mean score and standard errors of the described integration of breathing techniques in everyday life in the final questionnaire (Final) and the follow-up measurement after six months (Follow-up), $n=32 / 26$. 


\section{Discussion}

Since yoga promotes a balanced development of the physical, mental, and spiritual well-being of human beings, yogic courses have become one of the most sought-after courses around the world. An increasing number of studies are being conducted to understand the effectiveness of various yogic techniques, which further validates the growing interest of society in its benefits. While many engage in yoga for physical exercise and "simple" relaxation, its utilization beyond these aspects remains undiscovered. Apart from this, some people have a critical view of relaxation techniques, mindfulness, and the perceived spiritual and meditative aspects of yoga. As breathing techniques are the main component of different yogic practices, in this research, we studied the effectiveness of these techniques on a psychological level in a short-term intervention; they can be used as a support in everyday life and, in the future, as a complementary self-regulatory tool in psychotherapeutic treatment.

The goal of this study was to evaluate a breathing-based toolbox for self-regulation in everyday life. While not all hypotheses were confirmed, specific patterns of effects emerged for the individual breathing techniques, which were not seen in the control data of the baseline measurements. Ujjayi breathing showed a relaxing effect, reduced stress and tension, and increased peacefulness and being at ease. Paced breathing resulted in a greater awareness of inner experiences. Kapalabhati showed a significant increase in vitality and joy of life. In contrast, the alternate nostril breathing technique showed no hypothesis-compliant changes. However, compared to the previous intervention, subjects reported lower values for some scales, which was significant for vitality and joy of life.

Consistent with our first hypothesis, participants reported reduced levels of stress and tension and increased levels of peace and ease/leisure after practicing ujjayi for two weeks. It was also the technique participants found easiest to learn and was used most often in everyday life. Participants also described a higher accepting and non-judgmental attitude, which could be explained by the basic principles taught in the first intervention. In this intervention, the participants were given training on the perception of body and focus of breath with an attitude of mindfulness and acceptance, that is, without evaluation and interference. In order to practice this attitude, the first module already seemed sufficient, since the latter part consisting of the alternate nostril breathing technique did not result in any significant increase in accepting and non-judgmental attitude.

Practicing paced breathing was meant to enhance concentration. Participants described no significant changes in their ability to concentrate and react, but reported, in agreement with the hypothesis, an improvement in the awareness of inner experiences. It is possible that the audio files helped participants to train their focus with the auditory cue and connect it with the inner process of breathing. The lack of a general increase in concentration, except for the development of inner awareness, should be controlled with the evaluation of objective data. The development of inner awareness might indicate an increased capacity for focused attention and the associated enhancement of concentration.

The practice of kapalabhati, a mild form of hyperventilation, was supposed to activate and enhance participants' concentration. Data showed higher vitality and joy of life after practicing kapalabhati but no significant enhancement of concentration. However, this should be validated with an objective attention test, which could detect changes. 
Our fourth hypothesis predicted that alternate nostril breathing would have a balancing effect on the participant's arousal level, would lead to a higher accepting and non-judgmental attitude, and would result in higher satisfaction with the current state of the body. Self-report measures after the practice of this breathing technique showed a different picture than the measures after the other interventions. After two weeks of training and practicing alternate nostril breathing, participants reported reduced scores, such as a significant reduction in subjective vitality and joy of life. It seemed that compared to other interventions, this intervention might not have any specific benefit. It was also one of the more complicated interventions, and some participants had trouble during the training in closing one nostril as they felt that they were not getting enough air through the other nostril. Because of the specific hand position and visibility to the outside world, this technique was not incorporated well by the participants; it was rarely mentioned in the questionnaires neither in terms of the easiest (6.5\% of participants) nor the most difficult breathing technique (3.2\% of participants). It is possible that the wider, balancing effect of this breathing technique requires more practice and that the benefits of slow and regular breathing were already well perceived by the participants with the practice of ujjayi and paced breathing. The reduced vitality and joy of life could be caused by the reduced airflow through one nostril, which was reported by some participants.

In the final questionnaire, i.e., after the breathing training, participants reported a calming as well as a balancing effect. At the end of the training, participants integrated breathing techniques, especially ujjayi, into their everyday life. The other techniques were less often used in everyday life. After six months, the follow-up measurement revealed the same preference for ujjayi; the other techniques were not remembered that well. This may be because of the difficulty levels of other breathing techniques. Ujjayi is the simplest technique and was taught at the beginning of the training for all subjects. Because ujjayi interferes least with individuals' breathing rhythm, practicing it could have been comfortable. Denot-Ledunois, Vardon, Perruchet, and Gallego (1998) tested the effect of changes in breathing frequency and respiratory comfort. They found feelings of task difficulty to be correlated to respiratory comfort [28], in the way that the higher the task difficulty during voluntary controlled breathing (due to larger discrepancy from spontaneous pattern), the lesser the respiratory comfort would be. In our study, the participants may not have been comfortable with the new breathing technique because the time to deepen the technique and to practice them in everyday life between the interventions might have been too short.

These results suggest that the different breathing techniques could have different effects on the subjects. Therefore, they can be used as a tool for self-regulation, even in therapeutic contexts, and practiced every day. In psychotherapy, some single breathing techniques, e.g., slow breathing into the abdomen to reduce arousal during panic attacks, are already being used [29]. This can give patients increased feelings of safety and a sense of self-efficacy in dealing with their own level of arousal. Becoming aware of the breath and practicing breathing techniques could lead to a steady flow of breath, such as while breathing following a strict protocol to prolong the breath. It would be important to further investigate whether practicing these breathing techniques also leads to lower variability in everyday life. However, some effects of high variability have already been researched. There are some studies showing a connection between a high variability of breathing patterns and negatively associated states. Studies have indicated that patients with remitted depression exhibited higher variability in their breathing pattern during induced negative emotions compared to healthy controls [30]. Higher variability in breathing patterns was also related to lower daily life 
mood. While feeling emotions with high arousal, breathing pattern seems to change and be less regular [31], and studies have associated specific breathing patterns to basic emotions [32]. In combination with our study, it would be promising to study the different profiles of individual breathing techniques. One of the greatest advantages of the breath is that it is always available, and no additional materials are needed to use it. The initial teaching of the breathing techniques does not take much time, and the doubts regarding its usage in everyday life can be cleared by discussions. Therefore, by practicing these techniques, patients learn to regulate different mental states with different breathing techniques independently. It would offer new possibilities to use the breath not only for lowering vegetative arousal but also, for example, for increasing vitality and improving focus and self-awareness.

A limitation of the study is the small sample size. Also, due to the permuted design, carry-over effects may have occurred, which cannot be fully excluded. However, due to the complete permutation of the three interventions after ujjayi, carry-over effects can be expected to be less systematic compared to a design with a fixed sequence of interventions for all participants. In this design, a waiting control group could have been implemented via the different starting points of the three groups. Also, no comparison was made with training without respiratory focus. It is also important to analyze physiological data to verify the differential results of the questionnaires.

\section{Conclusions}

The results of the present study suggest differential effects for different breathing techniques. This could significantly expand the application of breathing - from an intervention used more in the relaxation domain toward a tool that can induce both relaxation and activation or promote awareness, depending on the need of the person. Further research is needed to validate the effects and to further understand the relationships between effect profiles and implemented breathing patterns. Also, the evaluation of physiological data is important to capture more objective measures to describe the effects.

\section{Acknowledgments}

We would like to thank the students that participated in the study and the BION team for their support, motivation and suggestions in the process of the study and writing.

\section{Author Contributions}

Janika Epe conceptualized and conducted training and research, and wrote the manuscript. Rudolf Stark helped designing the study and editing the manuscript. Ulrich Ott helped conceptualizing the training and edited the manuscript.

\section{Funding}

The research was funded by a grant from the behavioral therapy department of the Justus Liebig University Giessen. It was supported by the Bender Institute of Neuroimaging, Justus Liebig University Giessen. The preparation of the training materials was supported by the O.W. Barth press.

\section{Competing Interests}


Ulrich Ott and Janika Epe published a manual for the breathing training as book (in German) and receive royalties from the publishing house.

\section{References}

1. Matko K, Ott U, Sedlmeier P. What do meditators do when they meditate? Proposing a novel basis for future meditation research. Mindfulness. 2021; 12: 1791-1811.

2. Gerritsen RJ, Band GP. Breath of life: The respiratory vagal stimulation model of contemplative activity. Front Hum Neurosci. 2018; 12: 397.

3. Patañjali, Feuerstein G. The yoga-sūtra of Patañjali: A new translation and commentary. Rochester: Inner Traditions Internat; 1989.

4. Saoji AA, Raghavendra BR, Manjunath NK. Effects of yogic breath regulation: A narrative review of scientific evidence. J Ayurveda Integr Med. 2019; 10: 50-58.

5. Loew TH, Leinberger B, Hinterberger T. Entschleunigtes atmen. Psychother Dialog. 2017; 18: 6367.

6. Brown RP, Gerbarg PL. Sudarshan Kriya yogic breathing in the treatment of stress, anxiety, and depression: Part I-neurophysiologic model. J Altern Complement Med. 2005; 11: 189-201.

7. Everly GS, Lating JM. A clinical guide to the treatment of the human stress response. 3rd ed. New York: Springer New York; 2013.

8. Zaccaro A, Piarulli A, Laurino M, Garbella E, Menicucci D, Neri B, et al. How breath-control can change your life: A systematic review on psycho-physiological correlates of slow breathing. Front Hum Neurosci. 2018; 12: 353.

9. Stromberg SE, Russell ME, Carlson CR. Diaphragmatic breathing and its effectiveness for the management of motion sickness. Aerosp Med Hum Perform. 2015; 86: 452-457.

10. Shaffer F, McCraty R, Zerr CL. A healthy heart is not a metronome: An integrative review of the heart's anatomy and heart rate variability. Front Psychol. 2014; 5: 1040.

11. Lehrer PM, Gevirtz R. Heart rate variability biofeedback: How and why does it work? Front Psychol. 2014; 5: 756.

12. Stancák A, Kuna M, Srinivasan, Vishnudevananda S, Dostálek C. Kapalabhati--yogic cleansing exercise. I. Cardiovascular and respiratory changes. Homeost Health Dis. 1991; 33: 126-134.

13. Bischoff C, Straube A. Leitlinien klinische neurophysiologie. 1st ed. Stuttgart: Verlag W. Kolhammer; 2014.

14. Lys R. Zur Frage der aktivierenden wirkung der hyperventilation auf psychomotorische anfälle. Aktuelle Neurol. 1980; 7: 155-160.

15. Pal GK, Velkumary S, Madanmohan A. Effect of short-term practice of breathing exercises on autonomic functions in normal human volunteers. Indian J Med Res. 2004; 120: 115-121.

16. Sharma VK, Rajajeyakumar M, Velkumary S, Subramanian SK, Bhavanani AB, Madanmohan AS, et al. Effect of fast and slow pranayama practice on cognitive functions in healthy volunteers. J Clin Diagnostic Res. 2014; 8: 10-13.

17. Raghuraj $P$, Telles $S$. Immediate effect of specific nostril manipulating yoga breathing practices on autonomic and respiratory variables. Appl Psychophysiol Biofeedback. 2008; 33: 65-75.

18. Stančák Jr A, Kuna M. EEG changes during forced alternate nostril breathing. Int J Psychophysiol. 1994; 18: 75-79. 
19. Telles S, Verma S, Sharma SK, Gupta RK, Balkrishna A. Alternate-nostril yoga breathing reduced blood pressure while increasing performance in a vigilance test. Med Sci Monit Basic Res. 2017; 23: 392-398.

20. Thayer JF, Åhs F, Fredrikson M, Sollers III JJ, Wager TD. A meta-analysis of heart rate variability and neuroimaging studies: Implications for heart rate variability as a marker of stress and health. Neurosci Biobehav Rev. 2012; 36: 747-756.

21. Sedlmeier P, Eberth J, Schwarz M, Zimmermann D, Haarig F, Jaeger S, et al. The psychological effects of meditation: A meta-analysis. Psychol Bull. 2012; 138: 1139.

22. Ott U, Epe J. Gesund durch Atmen: Ein Neurowissenschaftler erklärt die Heilkraft der bewussten Yoga-Atmung. München: O.W. Barth; 2018.

23. Park YJ, Park YB. Clinical utility of paced breathing as a concentration meditation practice. Complement Ther Med. 2012; 20: 393-399.

24. Bergomi C, Tschacher W, Kupper Z. Konstruktion und erste validierung eines fragebogens zur umfassenden erfassung von achtsamkeit. Diagnostica. 2014; 60: 111-125.

25. Fliege $H$, Rose $M$, Arck $P$, Walter OB, Kocalevent RD, Weber $C$, et al. The Perceived Stress Questionnaire (PSQ) reconsidered: Validation and reference values from different clinical and healthy adult samples. Psychosom Med. 2005; 67: 78-88.

26. Frank R. Therapieziel wohlbefinden. Berlin: Springer Berlin Heidelberg; 2011.

27. Krägeloh CU, Bergomi C, Siegert RJ, Medvedev ON. Response shift after a mindfulness-based intervention: Measurement invariance testing of the comprehensive inventory of mindfulness experiences. Mindfulness. 2018; 9: 212-220.

28. Denot-Ledunois S, Vardon G, Perruchet P, Gallego J. Effects of voluntary changes in breathing frequency on respiratory comfort. Biol Psychol. 1998; 49: 71-82.

29. Hansch D. Erfolgreich gegen depression und angst: Wirksame selbsthilfe-anleitungen schritt für schritt-fallbeispiele und konkrete tipps. 2nd ed. Berlin: Springer; 2014.

30. Zamoscik VE, Schmidt SN, Gerchen MF, Samsouris C, Timm C, Kuehner C, et al. Respiration pattern variability and related default mode network connectivity are altered in remitted depression. Psychol Med. 2018; 48: 2364-2374.

31. Vlemincx E, Van Diest I, Van den Bergh O. Emotion, sighing, and respiratory variability. Psychophysiology. 2015; 52: 657-666.

32. Rainville $P$, Bechara A, Naqvi N, Damasio AR. Basic emotions are associated with distinct patterns of cardiorespiratory activity. Int J Psychophysiol. 2006; 61: 5-18. 
OBM Integrative and Complementary Medicine 2021; 6(3), doi:10.21926/obm.icm.2103031

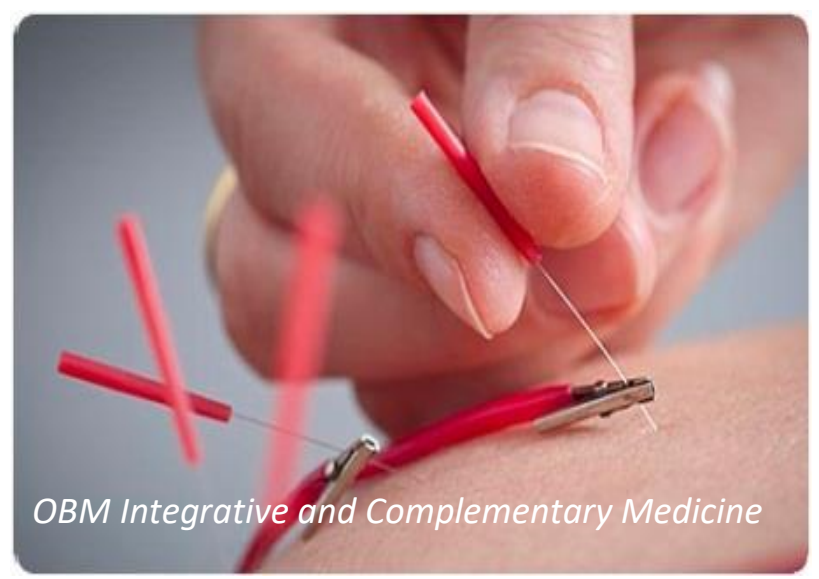

Enjoy $O B M$ Integrative and Complementary Medicine by:

1. Submitting a manuscript

2. Joining in volunteer reviewer bank

3. Joining Editorial Board

4. Guest editing a special issue

For more details, please visit:

http://www.lidsen.com/journals/icm 